\title{
Effect of manganese doping on the photoluminescence characteristics of chemically synthesized zinc sulfide nanoparticles
}

\author{
A. K. Kole $\cdot$ P. Kumbhakar
}

Received: 14 July 2011 / Accepted: 18 September 2011/Published online: 30 September 2011

(C) The Author(s) 2011. This article is published with open access at Springerlink.com

\begin{abstract}
The studies on luminescent II-VI semiconducting nanomaterials have attracted widespread attention recently, due to their potential applications in optoelectronic and biophotonic devices. Amongst other II-VI semiconductor nanoparticles (NPs), $\mathrm{Mn}^{2+}$-doped $\mathrm{ZnS}$ NPs having large exciton binding energy and wide direct band gap at room temperature have drawn considerable attention for exploring its interesting optical properties. However, in this report, water-soluble $\mathrm{Mn}^{2+}$-doped $\mathrm{ZnS}$ (ZnS:Mn) NPs with $\mathrm{Mn}^{2+}$ concentration varying between 1.5 and $5 \%$ (wt\%) have been synthesized by chemical co-precipitation method at room temperature. X-ray diffraction (XRD) studies and the analysis of the selected area electron diffraction (SAED) pattern, obtained from transmission electron microscopy (TEM), confirmed the formation of zinc blende structure in all the synthesized samples. The particle sizes of the samples, as obtained from the optical absorption studies, varies between 2.2 and $2.7 \mathrm{~nm}$ with the increase of $\mathrm{Mn}^{2+}$ concentration between 1.5 and $5 \%$. The room temperature photoluminescence (PL) emission measurements revealed the presence of yellow-orange emission band in all the $\mathrm{Mn}^{2+}$-doped samples which is attributed to $\mathrm{Mn}$ incorporation in $\mathrm{ZnS}$. The Gaussian fittings of the measured PL spectra of all the samples show the presence of four PL peaks. Amongst the four PL peaks three peaks appeared at 445, 476, and $520 \mathrm{~nm}$ in all the samples but the fourth yellow-orange emission peak suffered a red shift from 593 to $600 \mathrm{~nm}$ with increasing $\mathrm{Mn}^{2+}$ concentration from 1.5 to $5 \%$. In this report no quenching of yellow-
\end{abstract}

A. K. Kole · P. Kumbhakar $(\varangle)$

Nanoscience Laboratory, Department of Physics, National Institute of Technology, Durgapur 713209, West Bengal, India e-mail: pathik.kumbhakar@phy.nitdgp.ac.in;

nitdgpkumbhakar@yahoo.com orange emission peak is observed up to $5 \% \mathrm{Mn}^{2+}$ doping concentration in $\mathrm{ZnS}$. The synthesized water-soluble $\mathrm{ZnS}$ :Mn NPs can be further functionalized for using them as biolabels.

Keywords II-VI semiconductor · Nanostructures · Optical properties $\cdot$ Photoluminescence

\section{Introduction}

Nowadays extensive studies are carried out on synthesis and characterizations of optical properties of luminescent semiconductor nanomaterials because of their potential applications in optoelectronic devices, light emitters, detectors and solar cells (Nakada et al. 1999; Akihito et al. 2000; Yang et al. 2003; Fang et al. 2004; Fayaz et al. 2010). For the last few years the investigation has been focused on the preparation of II-VI semiconductor nanoparticles (NPs) for applications in the biological field as molecular probes or biolabels (Goldman et al. 2002). Semiconductor nanocrystallites have different optical and electronic properties than bulk materials. With decreasing particle size, 'quantum confinement effect' can be observed leading to a blue shift in the absorption spectrum of the particles (Brus 1998). Amongst others, $\mathrm{ZnS}$ is an II-IV semiconductor material and has a well-known wide direct band gap (3.71 eV at room temperature) and high exciton binding energy ( $40 \mathrm{meV}$ ) (Brus 1998; Bhargava et al. 1994; Bhargava 1996; Sapra et al. 2005). It is therefore a potential candidate for optoelectronic applications in the short wavelength region (green, blue, UV) of the electromagnetic spectrum. It is also a phosphor material with characteristic photoluminescent and electroluminescent properties that are utilized in the fields of lasers, sensors 
and displays. Luminescence properties of impurity-added $\mathrm{ZnS}$ NPs are widely different from those of bulk $\mathrm{ZnS}$. Nanocrystallites doped with optically active colour center provide new opportunities for luminescent study and applications (Fang et al. 2004; Peng et al. 2006; Son et al. 2007; Tiwari et al. 2010; Karar et al. 2004; Chattopadhyay et al. 2009a, b). Doping of rare earth ions in II-VI semiconductor is not favorable due to dissimilar chemical properties between the rare earth ions and host $\mathrm{Cd}^{2+}, \mathrm{Zn}^{2+}$ cations. However, the chemical properties of $\mathrm{Mn}^{2+}, \mathrm{Co}^{2+}$ and $\mathrm{Cu}^{2+}$ are very similar to those of $\mathrm{Cd}^{2+}$ or $\mathrm{Zn}^{2+}$ and thus $\mathrm{Mn}^{2+}$ can be easily doped with II-VI $\mathrm{ZnS}$ semiconductor host. The $\mathrm{Mn}^{2+}$-doped $\mathrm{ZnS}$ (i.e., $\mathrm{ZnS}: \mathrm{Mn}$ ) nanocrystals have been widely investigated since the first report of efficient luminescence emission in the yellow-orange region by Bhargava et al. (1994). In undoped II-VI semiconductors (e.g., CdS, CdSe, and $\mathrm{ZnS}$ ), the band gap can be tuned by controlling the particle sizes (Yang et al. 2003; Peng et al. 2006; Hu and Zhang 2006; Sarkar et al. 2008). Based on the report of (Bhargava et al. 1994; Bhargava 1996), research studies are carried out on doped II-VI semiconductor nanomaterials to enhance their light emission properties and thereby making them good candidate for optoelectronic applications (Fang et al. 2004; Peng et al. 2006; Hu and Zhang 2006; Kripal et al. 2010; Dong et al. 2010; Lu et al. 2010). Recently $\mathrm{Mn}^{+2}$-doped $\mathrm{ZnS}$ nanocrystals ( $\mathrm{ZnS}: \mathrm{Mn})$ have attracted much more attention because it is found that these materials can be used as efficient phosphor and in bio-imaging applications due to their nonlinear optical multiphoton absorption characteristics in the infrared and visible wavelength regions $(\mathrm{Hu}$ and Zhang 2006; Chattopadhyay et al. 2009a, b; Nazerdeylami et al. 2011).

The $\mathrm{Mn}^{2+}$ ion has a $d^{5}$ configuration and $\mathrm{Mn}^{2+} d$-electron states act as efficient luminescent centers while interacting strongly with $s$ - $p$ electronic states of the $\mathrm{ZnS}$ host into which external electronic excitation is normally directed. The subsequent transfer of electron and hole pairs into the electronic level of the $\mathrm{Mn}^{2+}$ ion leads to the characteristic yellow-orange light emission from the $\mathrm{Mn}^{2+}{ }^{4} \mathrm{~T}_{1}-{ }^{6} \mathrm{~A}_{1}$ transition ( $\mathrm{Lu}$ et al. 2001). Bhargava et al. (1994); Bhargava (1996) reported that, if the particle size is decreased, a strong hybridization of the $s$ $p$ states of the $\mathrm{ZnS}$ host and the $d$ states of the $\mathrm{Mn}^{2+}$ impurity should occur. Different possible mechanisms for the excitation of $\mathrm{Mn}^{2+}$ in $\mathrm{ZnS}$ host have been prescribed (Son et al. 2007; Hu and Zhang 2006). Various approaches have been reported in the recent past to improve the luminescent properties of doped II-VI semiconductors. Recently, it has also been reported that temperature, $\mathrm{pH}$ of the solution, and the solvent used during synthesis play crucial roles in NPs growth.

However, for applications of NPs in biological field, it is desirable that the prepared semiconductor NPs should be water soluble. In this work, water-soluble $\mathrm{Mn}^{2+}$-doped $\mathrm{ZnS}$
NPs have been synthesized using a simple chemical coprecipitation method. The chemical co-precipitation method is most popular synthesis mechanism due to its various advantages, such as it is more economical than the other synthesis methods, doping of foreign atoms are easily possible as well as less instrumentation are required (Sarkar et al. 2008). The effect of $\mathrm{Mn}^{2+}$ doping on the photoluminescence (PL) property of chemically synthesized ZnS NPs is also studied in the present work. The size of the synthesized $\mathrm{ZnS}$ :Mn NPs lie in $\sim 2.2-2.7 \mathrm{~nm}$ range. It is found in this work that with increasing $\mathrm{Mn}^{2+}$ concentration the average particle sizes of the synthesized samples are increased. A blue shift in the UV-visible optical absorption spectra of the prepared $\mathrm{ZnS}: \mathrm{Mn}$ nanocrystals are observed with respect to that of bulk $\mathrm{ZnS}$ and this has occurred as a result of 'quantum confinement effect' (Brus 1998). We have also recorded PL emission spectra from the synthesized samples at room temperature. Different groups have reported previously the $\mathrm{PL}$ emission characteristics of $\mathrm{ZnS}$ and $\mathrm{ZnS}$ :Mn nanostructures with different crystalline structures and observed blue, green and yellow-orange light emissions and prescribed different origin for such emissions (Bhargava et al. 1994, Son et al. 2007; Tiwari et al. 2010; Hu and Zhang 2006; Sarkar et al. 2008; Lu et al. 2001; Sooklal et al. 1996). The simultaneous presence of the yellow-orange PL peak along with the PL emission in the blue region in $\mathrm{Mn}^{2+}$-doped $\mathrm{ZnS}$ samples confirm the $\mathrm{Mn}^{2+}$ incorporation in the synthesized $\mathrm{ZnS}$ NPs (Sooklal et al. 1996). Further, it is found by Gaussian fittings of the measured PL spectra of our samples that there exist three peaks at 445, 476, and $520 \mathrm{~nm}$ in all the samples but a red shift from 593 to $600 \mathrm{~nm}$ is observed for the fourth yellow-orange emission peak with increasing $\mathrm{Mn}^{2+}$ concentration from 1.5 to 5\%. Many researchers (Cao et al. 2009; Xiao and Xiao 2008; Peng et al. 2005; Murugadoss et al. 2010) found concentration quenching for the photoluminescence intensity of yellow-orange emission peak of $\mathrm{Mn}^{2+}$-doped $\mathrm{ZnS}$ nanocrystals at a lower concentration of $\mathrm{Mn}^{2+}$. However, in this report no quenching of yelloworange PL emission has been observed up to $5 \% \mathrm{Mn}^{2+}$ doping in the synthesized ZnS:Mn NPs. The intensity of the fourth PL peak has been found to be enhanced with the increasing $\mathrm{Mn}^{2+}$ concentration, whereas the same for other three peaks remains almost unaltered. The synthesized NPs can be used in different biomedical applications after functionalizing them with other suitable materials to make the NPs bioactive as well as to retain their shape and size (Goldman et al. 2002).

\section{Experiment}

$\mathrm{Mn}^{2+}$-doped $\mathrm{ZnS}$ NPs are synthesized at room temperature in distilled water medium by chemical co-precipitation 
method, which is described below. All the chemicals used are of AR grade (Merck and SD fine chemicals) and they are used without further purification. $\mathrm{Mn}^{2+}$-doped (1.5-5.0 wt\%) ZnS NPs (ZnS:Mn) are prepared by mixing calculated amounts of zinc nitrate solution in distilled water and manganese acetate solution in distilled water under continuous stirring followed by drop-wise addition of sodium sulfide solution in distilled water up to $\mathrm{pH}$ 8. The mixture is continuously stirred in a magnetic stirrer for another $1 \mathrm{~h}$. After $1 \mathrm{~h}$ of continuous stirring, a yellowishwhite precipitate is deposited at the bottom of the flask. The precipitate is separated from the reaction mixture by centrifugation (Eltek Refrigerated Centrifuge RC4100D) for $10 \mathrm{~min}$ at $5,000 \mathrm{rpm}$ and washed several times with distilled water and methanol to remove the remaining sodium particles, if any. The wet precipitate is then dried for further measurements. Four different samples those are synthesized; henceforth will be called as S1, S2, S3, and S4 corresponding to the $\mathrm{ZnS}: \mathrm{Mn}$ (1.5\%), $\mathrm{ZnS}: \mathrm{Mn}$ (2.5\%), ZnS:Mn (3.5\%), and ZnS:Mn (5.0\%), respectively.

The optical transmission spectra of the prepared ZnS:Mn NPs dispersed in water are recorded using a UVvisible spectrophotometer (Hitachi, U-3010). The formation of $\mathrm{ZnS}: \mathrm{Mn}$ NPs have been confirmed by transmission electron microscopy (TEM, JEOL 2000 FX-II). The XRD pattern is recorded by using an X-ray diffractometer (PANLYTICAL) with $\mathrm{Cu} \mathrm{K}_{\alpha}$ radiation of wavelength $\lambda=0.15406 \mathrm{~nm}$ in the scan range $2 \theta=20^{\circ}-70^{\circ}$. The photoluminescence (PL) spectra of the ZnS:Mn NPs dispersed in water are recorded using a spectrofluorimeter (Perkin Elmer LS-55).

\section{Results and discussion}

Figure 1 shows the UV-visible absorption characteristics of all the synthesized samples. To obtain the absorption characteristics of all the samples, at first transmittance $(T)$ at different wavelengths $(\lambda)$ are measured and then absorbance $(\alpha)$ at the corresponding wavelengths are calculated by using Beer-Lambert relation:

$\alpha=\frac{1}{d} \ln \left(\frac{1}{T}\right)$,

where $d$ is the path length. The linear optical transmission characteristics of the prepared samples are measured by at first dispersing the powdered samples in water and then it is taken in a quartz cuvette of $10 \mathrm{~mm}$ path length $(d)$. As shown in Fig. 1 the absorption onsets of the samples are fairly blue-shifted from the absorption band-edge of $334 \mathrm{~nm}$ (shown as a vertical line in Fig. 1) of the bulk $\mathrm{ZnS}$. Such blue shift in the band-edge takes place due to 'quantum confinement effect' (Brus 1998). The absorption

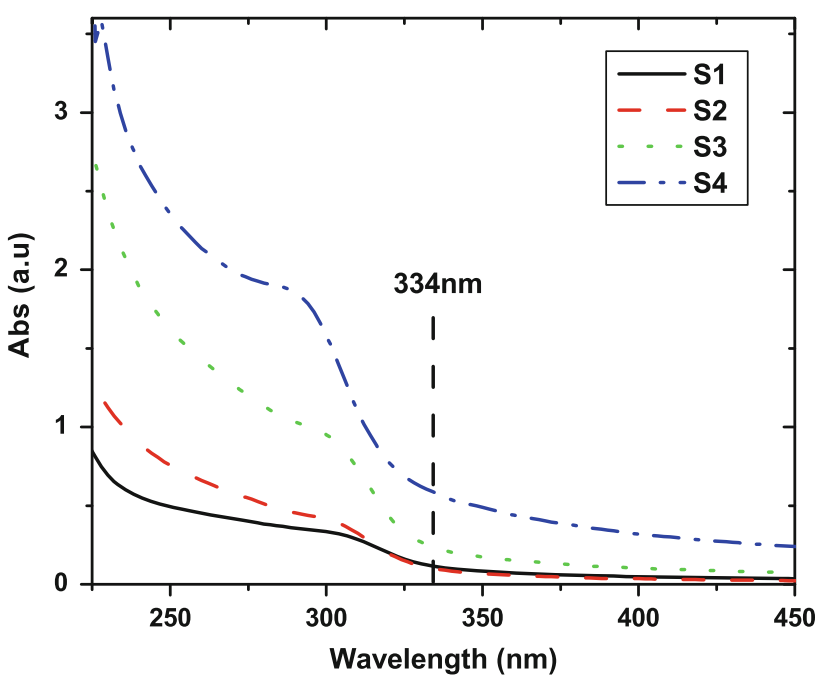

Fig. 1 UV-visible absorption characteristic of the prepared ZnS:Mn nanoparticles dispersed in distilled water. The vertical line shows the absorption band-edge of bulk ZnS. Here, S1, S2, S3, and S4 corresponds to the $\mathrm{ZnS}: \mathrm{Mn}$ (1.5\%), ZnS:Mn (2.5\%), ZnS:Mn (3.5\%), and $\mathrm{ZnS}: \mathrm{Mn}(5.0 \%)$ samples, respectively

edges of the samples are broad due to the distribution of particle sizes in the samples. The absorption coefficient $\alpha$ and band gap $E_{g n}$ of a semiconductor material can be related as,

$(\alpha h v)^{m}=A\left(h v-E_{g n}\right)$

where $A$ is a constant and the band gap of the studied material is denoted as $E_{g n}$. Exponent " $m$ " depends upon the type of the transition: $m$ may have values $2,1 / 2,2 / 3$ and $1 / 3$ corresponding to the allowed direct, allowed indirect, forbidden direct and forbidden indirect transitions, respectively (Pankove 1971). For $\mathrm{ZnS}$, the value of $m$ is always 2, i.e., the fundamental absorption corresponds to allowed direct transition. To calculate the band gap values of the prepared samples, $(\alpha h v)^{2}$ versus $h v$ has been plotted and it is shown in Fig. 2. The value of the band gap is determined by extrapolating the straight line portion of $(\alpha h v)^{2}$ versus $h v$ graph to the $h v$ axis. The calculated values of band gaps are 5.6, 5.5, 5.1, and $5.0 \mathrm{eV}$, respectively, for S1, S2, S3, and S4 samples. It is found that the calculated values of band gap of all the synthesized samples are higher than that of bulk $\mathrm{ZnS}$. The shift of the band gap of the synthesized samples from the bulk value of $3.71 \mathrm{eV}$ (334 nm) of $\mathrm{ZnS}$ is observed due to quantum confinement effect.

According to quantum confinement theory, electrons in the conduction band and holes in the valence band are spatially confined by the potential barrier of the surface. Due to confinement of both electrons and holes, the lowest energy optical transition from the valence to conduction band will increase in energy, effectively increasing the bandgap, as described by the following equation: 


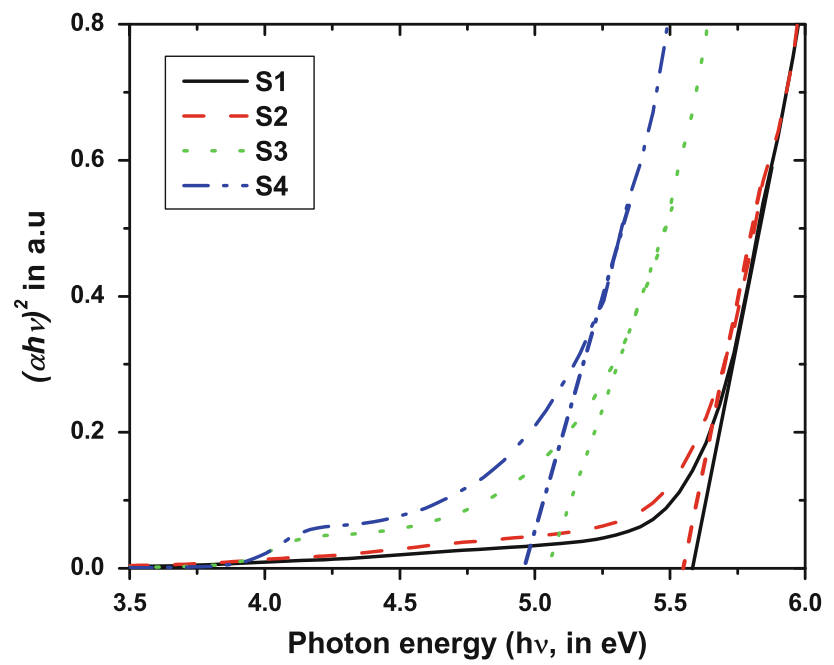

Fig. 2 The plot of $(\alpha h v)^{2}$ versus $h v$ of $\mathrm{ZnS}: \mathrm{Mn}$ nanoparticles. Here, $S 1, S 2, S 3$, and $S 4$ corresponding to the $\mathrm{ZnS}: \mathrm{Mn}$ (1.5\%), ZnS:Mn (2.5\%), ZnS:Mn (3.5\%), and ZnS:Mn (5.0\%), respectively

$E_{g n}=E_{g}+h^{2} \pi^{2} / 2 \mu r^{2}$,

where $E_{g}$ is the band gap of the bulk semiconductor, $E_{g n}$ is band gap of the synthesized ZnS:Mn NPs and $h$ is Planck's constant, $r$ is the radius of the NPs, and $\mu$ is the reduced mass of the exciton given by $\mu=m_{\mathrm{e}}^{*} m_{\mathrm{h}}^{*} / m_{\mathrm{e}}^{*}+m_{\mathrm{h}}^{*}$. Here, $m_{\mathrm{e}}^{*}$ and $m_{\mathrm{h}}^{*}$ are effective masses of the electron and hole, respectively. This model was expanded by Brus (1998) to include Coulombic interaction of excitons and the correlation energy and can be written as:

$E_{g n}=E_{g b}+h^{2} \pi^{2} / 2 \mu r^{2}-1.786 e^{2} / \varepsilon r+0.284 E_{\mathrm{R}}$

Here, $E_{\mathrm{R}}$ is the Rydberg (spatial correlation) energy and $\varepsilon$ is the dielectric constant of the bulk semiconductor. Hence, the UV transmission band-edge of NPs will shift to higher frequency with decreasing particle diameter, with a dependence on $1 / r^{2}$. Neglecting the polarization term and by substituting $m_{\mathrm{e}}^{*}=0.34 m_{o}, m_{\mathrm{h}}^{*}=0.23 m_{o}, m_{o}$ is the free electron mass, relative dielectric constant $(\varepsilon)$ of $\mathrm{ZnS}=8.76$, in Eq. 4, the band gap of ZnS NPs with radius $r(\mathrm{~nm})$ can be given as:

$E_{g n}=E_{g}+2.7442 / r^{2}-0.2963 / r$.

From the calculated band gap as described above and by using Eq. 5, the average size (diameter) $\mathrm{Mn}^{2+}$-doped samples have been calculated and those are summarized in Table 1 along with some other data. The calculated values from optical absorption studies matches well with those obtained from transmission electron microscope (TEM) micrograph analyses as described below.

We can get direct information about particle size and morphology of the prepared nanocrystalline samples by analyzing TEM micrograph of the samples. However, a
Table 1 Particle sizes of all the synthesized samples from optical studies and interplanar spacing $\left(d_{h k l}\right)$ from XRD and JCPDS data card and corresponding ( $h k l$ ) values

\begin{tabular}{|c|c|c|c|c|c|}
\hline $\begin{array}{l}\text { Name } \\
\text { of the } \\
\text { sample }\end{array}$ & $\begin{array}{l}\mathrm{Mn}^{2+} \\
\text { concentration } \\
(\%)\end{array}$ & $\begin{array}{l}\text { Particle size from } \\
\text { optical studies } \\
\text { (nm) }\end{array}$ & $(h k l)$ & $\begin{array}{l}d_{X R D} \\
(\AA)\end{array}$ & $\begin{array}{l}d_{J C P D F} \\
(\AA)\end{array}$ \\
\hline \multirow[t]{3}{*}{ S1 } & 1.5 & 2.2 & (111) & 3.11 & 3.10 \\
\hline & & & $(220)$ & 1.89 & 1.89 \\
\hline & & & (311) & 1.62 & 1.61 \\
\hline \multirow[t]{3}{*}{ S2 } & 2.5 & 2.3 & (111) & 3.13 & 3.10 \\
\hline & & & (220) & 1.89 & 1.89 \\
\hline & & & (311) & 1.62 & 1.61 \\
\hline \multirow[t]{3}{*}{ S3 } & 3.5 & 2.6 & (111) & 3.11 & 3.10 \\
\hline & & & $(220)$ & 1.89 & 1.89 \\
\hline & & & (311) & 1.62 & 1.61 \\
\hline \multirow[t]{3}{*}{ S4 } & 5.0 & 2.7 & (111) & 3.10 & 3.10 \\
\hline & & & $(220)$ & 1.89 & 1.89 \\
\hline & & & (311) & 1.62 & 1.61 \\
\hline
\end{tabular}

typical TEM image of $2.5 \% \mathrm{ZnS}: \mathrm{Mn}$ sample is shown in Fig. $3 \mathrm{a}$ and its inset shows the corresponding selected area electron diffraction (SAED) pattern. From Fig. 3a it is seen that particles in various sizes are present in the prepared samples. As the particle size distribution is asymmetric, in order to get average particle size, the particle size distribution is fitted with a log-normal distribution which is shown in Fig. 3b. The calculated average particle size from TEM image analysis is about $2.5 \mathrm{~nm}$ which matches well with the value of the same as obtained from optical studies and XRD analysis and is described below. Three circular rings that appeared in SAED pattern are assigned to (111), (220) and (311) crystalline planes of cubic ZnS.

Figure 4 shows the XRD patterns of all the prepared ZnS:Mn samples. The three diffraction peaks appeared with $2 \theta$ values of $28.70^{\circ}, 47.96^{\circ}$, and $56.76^{\circ}$ corresponding to the (111), (220) and (311) planes, respectively, of the cubic phase (zinc blende) ZnS matching JCPDS 80-020. No phase transformation due to $\mathrm{Mn}^{2+}$ doping has been observed in all the synthesized samples. Similar result has also been reported previously by Karar et al. (2004). As shown in Fig. 4 the XRD peaks are broadened due to nanocrystalline nature of the synthesized samples. From the value of full width at half maximum (FWHM) of the most prominent peak in XRD pattern, the mean crystallite sizes are calculated by using the Debye-Scherer's formula (Cullity 1978):

$D=0.89 \lambda /(\beta \cos \theta)$.

where $D$ is the average crystallite size, $\lambda$ is the $\mathrm{X}$-ray wavelength, $\theta$ is the diffraction angle and $\beta$ is the FWHM of the most prominent diffraction peak. The calculated average crystallite sizes from XRD data, viz. for $2.5 \%$ 

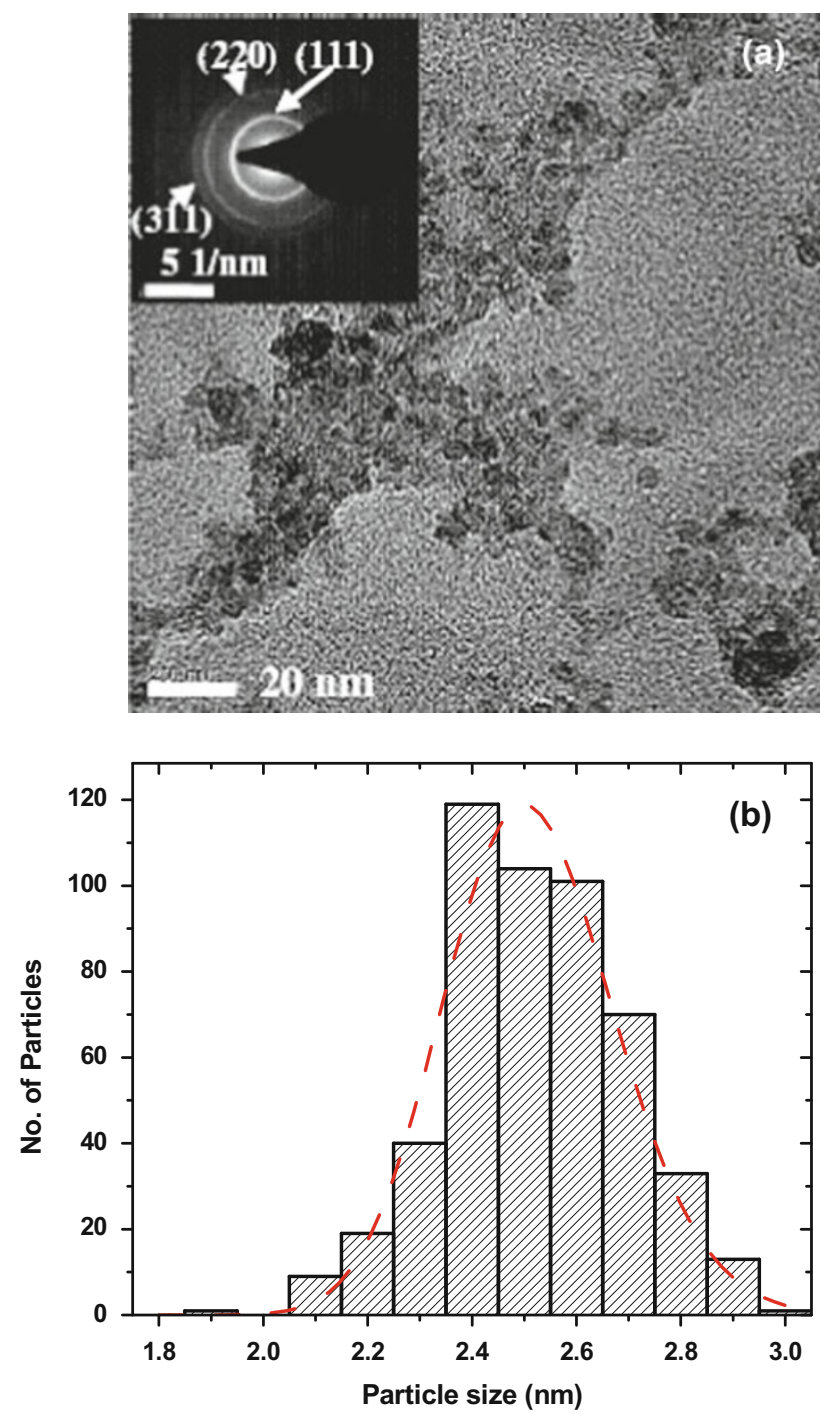

Fig. 3 a Shows the TEM image of $2.5 \% \mathrm{Mn}^{2+}$-doped $\mathrm{ZnS}$ sample and its inset shows the SAED pattern which confirms the diffraction from (111), (220) and (311) planes of cubic ZnS. b Shows the particle size distribution obtained from TEM image of $2.5 \% \mathrm{Mn}^{2+}$-doped $\mathrm{ZnS}$ sample. The distribution is fitted with log-normal distribution

$\mathrm{ZnS}: \mathrm{Mn}$ sample is $2.4 \mathrm{~nm}$ which agrees well with the values as calculated from optical studies and TEM measurements. The interplanar spacing $\left(d_{h k l}\right)$ and corresponding $(h k l)$ values for $\mathrm{S} 1, \mathrm{~S} 2, \mathrm{~S} 3$, and $\mathrm{S} 4$ samples as calculated from XRD and JCPDS data cards are summarized in Table 1.

The photoluminescence emission (PL) spectra for the S1, S2, S3, and S4 samples are shown in Fig. 5a. To measure PL emission spectra, samples are dispersed in water and taken in a quartz cuvette of $10 \mathrm{~mm}$ path length. Different excitation wavelengths are used but the maximum emission intensity is observed when the excitation wavelength of $330 \mathrm{~nm}$ is used for all the samples. From the Fig. 5a it is clear that the PL emission band obtained from

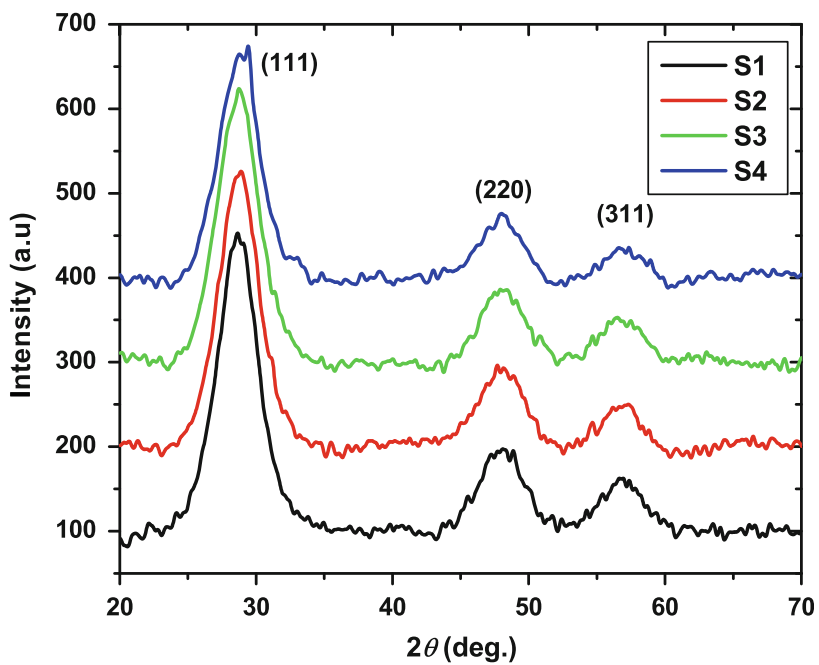

Fig. 4 X-ray diffraction (XRD) pattern of all the ZnS:Mn samples. All the curves have been scaled to plot together. Here, S1, S2, S3, and S4 corresponding to the $\mathrm{ZnS}: \mathrm{Mn}$ (1.5\%), ZnS:Mn (2.5\%), ZnS:Mn (3.5\%), and $\mathrm{ZnS}: \mathrm{Mn}(5.0 \%)$, respectively

the different $\mathrm{ZnS}: \mathrm{Mn}$ samples are highly asymmetrical and broadened with multiple peaks indicating the involvement of different luminescence centers in the radiative processes.

The Gaussian fittings of the measured PL spectra of S1, S2, S3, and S4 samples are shown in Fig. 5b, c, d, e, respectively. In all the figures dashed curves (red color) show the theoretical Gaussian fitting of experimentally obtained curves (solid lines) and the dotted curves (green color) show the individual Gaussian curves. From the Fig. 5b-e, it is found that PL spectra of all the samples contain four PL peaks lying in the visible region of the electromagnetic spectrum. Amongst the four peaks three peaks appear at 445, 476, and $520 \mathrm{~nm}$ irrespective of the sample but the fourth peak which appears in the yelloworange region suffers a red shift from 593 to $600 \mathrm{~nm}$ with increasing $\mathrm{Mn}^{2+}$ concentration from 1.5 to $5 \%$. Different groups have reported the PL emission from $\mathrm{ZnS}$ nanostructures with different morphologies, including nanorods, nanowires, and nanobelts, etc. (Fang et al. 2004). There are various reports of near band-edge PL emission at $340 \mathrm{~nm}$, a self-activated PL band at $420 \mathrm{~nm}$, as well as a green PL band at $\sim 535 \mathrm{~nm}$ (Ye et al. 2004). Karar et al. (2004) have reported synthesis of ZnS:Mn NPs with Mn concentration varied up to $40 \%$ by chemical method using polyvinyl pyrrolidone (PVP) as a capping agent and iso-propanol as the solvent. They have reported PL emission fitted with four Gaussian peaks at 460, 600, 640 and $680 \mathrm{~nm}$. Green PL emission from ZnS NPs at $\sim 535 \mathrm{~nm}$ is reported by Ye et al. (2004) and it is assigned to elemental sulfur species present in the samples. Sapra et al. (2005) have reported synthesis of ZnS:Mn NPs with DMF as the solvent and 1-thioglycerol as the capping agent and they have reported 

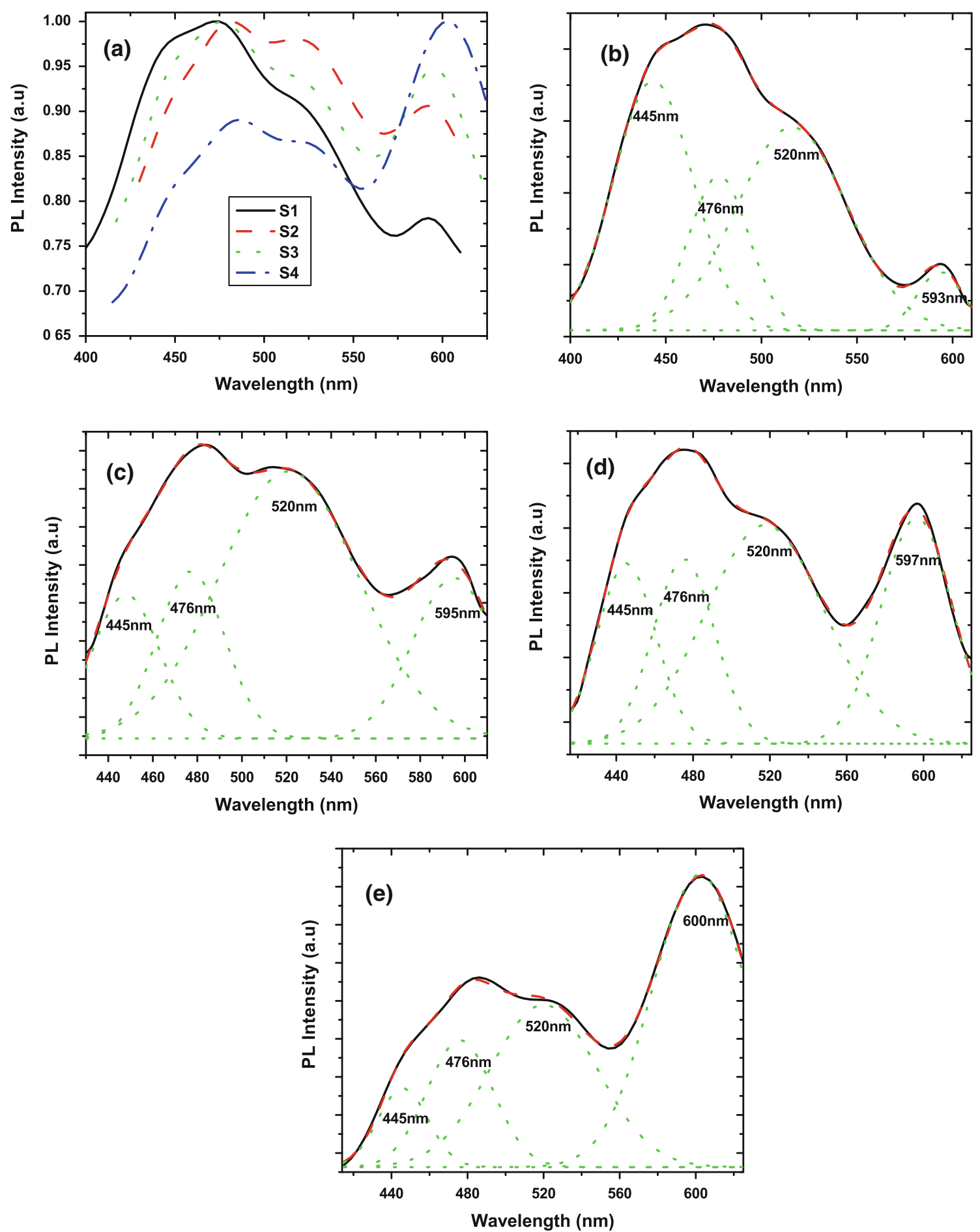

Fig. 5 a Room temperature photoluminescence $(P L)$ spectra of all ZnS:Mn samples. The Gaussian fittings of individual PL spectra of ZnS:Mn (1.5\%), ZnS:Mn (2.5\%), ZnS:Mn (3.5\%) and ZnS:Mn $(5.0 \%)$ samples are shown in $\mathbf{b}$, c, $\mathbf{d}$, and e, respectively. The

PL emission at $425 \mathrm{~nm}$ from $\mathrm{ZnS}$ due to de-excitation of defect states and $580 \mathrm{~nm}$ PL emission from $\mathrm{ZnS}: \mathrm{Mn}$ samples due to Mn $d-d$ transitions. Son et al. (2007) have reported synthesis of ZnS:Mn NPs with average sizes $7.3 \mathrm{~nm}$ by liquid-solid solution (LSS) method by excitation wavelength used for all the samples is $330 \mathrm{~nm}$. In all the figures dashed curves (red color) show the theoretical Gaussian fitting of experimentally obtained curves and the dotted curves (green color) show the individual Gaussian curves

employing suitable organic agents. They have reported PL emission from the samples at $580 \mathrm{~nm}$.

However, the well-known blue emission peak observed at $445 \mathrm{~nm}$ in our samples is associated with the defectrelated emission of the $\mathrm{ZnS}$ host, the peak at $476 \mathrm{~nm}$ is 
attributed to sulfur bonds dangling at the interface of $\mathrm{ZnS}$ NPs and the green PL peak at $520 \mathrm{~nm}$ observed in this work is assigned to elemental sulfur species on the surface of $\mathrm{ZnS}$ (Tiwari et al. 2010; Denzler et al. 1998). The origin of the fourth PL peak in our samples is due to $\mathrm{Mn}^{2+}$ incorporation in the $\mathrm{ZnS}$ host lattice. It was reported by Sooklal et al. (1996) that in $\mathrm{Mn}^{2+}$-activated $\mathrm{ZnS}$ nanocrystals in which the $\mathrm{Mn}^{2+}$ ions are distributed outside the $\mathrm{ZnS}$ nanocrystals, the PL is totally different from that of $\mathrm{Mn}^{2+}$-doped $\mathrm{ZnS}$ nanocrystals in which the $\mathrm{Mn}^{2+}$ is incorporated within the nanocrystals. When the $\mathrm{Mn}^{2+}$ is incorporated within the nanocrystals, both the $435 \mathrm{~nm}$ blue emission of $\mathrm{ZnS}$ and the orange $\mathrm{Mn}^{2+}$ emission at $590 \mathrm{~nm}$ are observed. However, in the $\mathrm{Mn}^{2+}$-activated $\mathrm{ZnS}$ nanocrystals in which the $\mathrm{Mn}^{2+}$ ions are distributed outside the $\mathrm{ZnS}$ nanocrystals, no orange emission at $590 \mathrm{~nm}$ is observed; a new peak at $350 \mathrm{~nm}$ appears and the blue $435 \mathrm{~nm}$ emission of $\mathrm{ZnS}$ is quenched considerably and shifted to $390 \mathrm{~nm}$ (Sooklal et al. 1996). When $\mathrm{Mn}^{2+}$ ions are incorporated into the $\mathrm{ZnS}$ lattice and substitute for host cation sites; the hybrid between the $s-p$ electrons of the host $\mathrm{ZnS}$ and the $d$ electrons of $\mathrm{Mn}^{2+}$ occurs and makes the forbidden transition of ${ }^{4} \mathrm{~T}_{1}-{ }^{6} \mathrm{~A}_{1}$ partially allowed, as a result the yellow-orange $\mathrm{PL}$ originates from a transition between the ${ }^{4} \mathrm{~T}_{1}$ excited state and the ${ }^{6} \mathrm{~A}_{1}$ ground state of the $\mathrm{Mn}^{2+}$ ion within a nanocrystalline $\mathrm{ZnS}$ lattice (Bhargava et al. 1994; Denzler et al. 1998; Ren et al. 2008; Cao et al. 2009). Therefore, by comparing these studies and our results it is concluded that in our synthesized samples $\mathrm{Mn}^{2+}$ ions are incorporated within the ZnS NPs.

From Fig. 6 it is also observed that the position of the fourth PL peak has been red-shifted gradually from 593 to $600 \mathrm{~nm}$ with the increase in the $\mathrm{Mn}^{2+}$ concentration in the synthesized samples. The models involving a size-dependent phonon coupling and crystal fields can be used for an explanation for the observed PL peak shift in the present study (Yang et al. 2003; Xiao and Xiao 2008; Peng et al. 2005; Murugadoss et al. 2010; Pradhan et al. 2005; Chen et al. 2000). It has been reported that the $\mathrm{Mn}^{2+}$ emission band in ZnS NPs may shift to higher or to lower energies compared to bulk $\mathrm{ZnS}: \mathrm{Mn}^{2+}$, depending on both the size and the surface effects (Yang et al. 2003). The increase in particle size leads to red shift of the emission peak

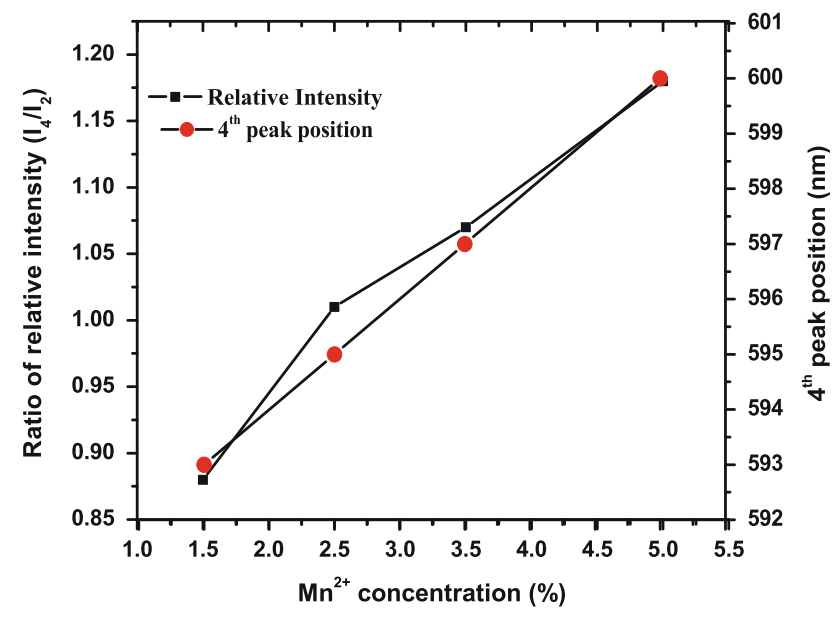

Fig. 6 Ratio of relative intensities $\left(I_{4} / I_{2}\right)$ and the position of the fourth PL peak $\left(I_{4}\right)$ with increasing $\mathrm{Mn}(\%)$ concentration

consistent with quantum size effect. The decrease in the density of surface states with increasing particle size can be attributed to the observed red shift in emission peak wavelength.

Many researchers have found concentration quenching for the PL intensity of yellow-orange emission peak of $\mathrm{Mn}^{2+}$-doped $\mathrm{ZnS}$ nanocrystals (Cao et al. 2009; Xiao and Xiao 2008; Peng et al. 2005; Murugadoss et al. 2010). Cao et al. (2009) have reported optimal doping concentration of $\mathrm{Mn}$ at 3\%. Xiao and Xiao (2008) showed a maximum luminescence at a doping concentration of $1.5 \%$, Peng et al. (2005) obtained a maximum luminescence at a doping concentration of $1.03 \%$. Very recently, Murugadoss et al. (2010) obtained a maximum luminescence at a doping concentration of $4 \%$. In this report, no quenching of yellow-orange PL emission is observed up to $5 \% \mathrm{Mn}$ doping in the synthesized $\mathrm{ZnS}: M n$ samples.

To understand the relative variation in the intensity of the fourth PL peak in comparison to the other peaks, the relative values of the intensity of the third and fourth PL peaks with respect to the second PL peak are calculated and these are summarized in Table 2. Also the variation of relative intensity $\left(I_{4} / I_{2}\right)$, with the $\mathrm{Mn}^{2+}$ concentration is shown in Fig. 6. Here $I_{4}$ is the intensity of the fourth PL peak and $I_{2}$ is the intensity of the second PL peak. From Fig. 6 it is found that with increasing $\mathrm{Mn}^{2+}$ concentration

Table 2 Photoluminescence (PL) emissions peak positions and their relative peak intensities

\begin{tabular}{lllllll}
\hline Sample & $\begin{array}{l}\text { First PL } \\
\text { peak }(\mathrm{nm})\end{array}$ & $\begin{array}{l}\text { Second PL } \\
\text { peak }(\mathrm{nm})\end{array}$ & $\begin{array}{l}\text { Third PL } \\
\text { peak }(\mathrm{nm})\end{array}$ & $\begin{array}{l}\text { Fourth PL } \\
\text { Peak (nm) }\end{array}$ & $\begin{array}{l}\text { Relative intensity of } \\
\text { the third peak with } \\
\text { respect to second peak }\end{array}$ & $\begin{array}{l}\text { Relative intensity of } \\
\text { the fourth peak with } \\
\text { respect to second peak }\end{array}$ \\
\hline S1 & 445 & 476 & 520 & 593 & 1.1 & 0.9 \\
S2 & 445 & 476 & 520 & 595 & 1.1 & 1.0 \\
S3 & 445 & 476 & 520 & 597 & 1.1 & 1.1 \\
S4 & 445 & 476 & 520 & 600 & 1.0 & 1.2 \\
\hline
\end{tabular}


(up to $5 \%$ ) the intensity of the yellow-orange peak which arises due to $\mathrm{Mn}^{2+}{ }^{4} \mathrm{~T}_{1}-{ }^{6} \mathrm{~A}_{1}$ transition increases as compared to the blue emission $(476 \mathrm{~nm})$ peak arising due to sulfur dangling bonds. This suggests that with increasing $\mathrm{Mn}^{2+}$ concentration, $\mathrm{Mn}^{2+}$ emission is growing and at the same time there is some loss of energy in the host lattice emission. Pradhan et al. (2005) have previously studied the possible nucleation and growth process, they found that the successful doping and decoupling of doping must fulfill following conditions: with the increase in doped ions, the steady increase in the PL intensity from the doping centers at about $605 \mathrm{~nm}$, the fixed PL positions of the host $\mathrm{ZnS}$ nanocrystals and the doping centers, and the gradual decrease in the PL intensity of the host $\mathrm{ZnS}$ nanocrystals. Generally, in doped semiconductors, there are two channels for luminescence excitation. One is indirect excitation, i.e., excitation into the excited levels of the host, followed by an energy transfer from the host to the impurity ions to cause the luminescence (Chen et al. 2000). Another is the direct excitation. In the present case indirect excitation at $330 \mathrm{~nm}$ has led to the yellow-orange PL emission from the synthesized samples. The successful observation of $\mathrm{Mn}^{2+}$ emission in $\mathrm{ZnS}$ NPs from indirect excitation at $330 \mathrm{~nm}$ proves that energy transfer from $\mathrm{ZnS}$ to $\mathrm{Mn}^{2+}$ is taking place and this is another proof showing that $\mathrm{Mn}^{2+}$ is located within the $\mathrm{ZnS}$ clusters.

\section{Conclusion}

$\mathrm{Mn}^{2+}$-doped $\mathrm{ZnS}$ (ZnS:Mn) NPs having 2.2-2.7 $\mathrm{nm}$ in sizes are synthesized by chemical precipitation methods at room temperature by using distilled water as solvent. The UV-visible absorption spectroscopy shows the strong confinement effect which suggests the formation of NPs. XRD studies revealed cubic phase of all the synthesized $\mathrm{ZnS}: \mathrm{Mn}$ samples. The PL spectra from all the samples are measured at room temperature and from the Gaussian fittings of the measured PL spectra of all the samples it is found that four PL peaks are present in the PL spectra of all the samples. Amongst them three PL peaks appeared at fixed positions of 445, 476, and $520 \mathrm{~nm}$ in all the samples but a red shift from 593 to $600 \mathrm{~nm}$ takes place for the fourth yellow-orange light emission peak with increasing $\mathrm{Mn}^{2+}$ concentration from 1.5 to $5 \%$. It is also found that the intensity of yellow-orange PL peak increases gradually relative to other peaks unlike the previous reports. The steady increase in the PL intensity of the yellow-orange peak with the increase in doped ions and the fixed PL peak positions of the host $\mathrm{ZnS}$ nanocrystals, and the gradual decrease in the PL intensity of the host $\mathrm{ZnS}$ nanocrystals confirms the doping of $\mathrm{Mn}^{2+}$ in $\mathrm{ZnS}$. The red shift of yellow-orange light emission peak with increasing $\mathrm{Mn}^{2+}$ concentration can be related to the particle size-dependent crystal field effect and the surface effects.

Out of various II-VI direct band gap semiconductor, $\mathrm{ZnS}$ as well as $\mathrm{Mn}^{2+}$-doped $\mathrm{ZnS}$ NPs has wide direct band gap, high refractive index and multiphoton absorption characteristics as studied by various researchers. $\mathrm{ZnS}$ :Mn NPs emit yellow-orange light when excited to UV radiation and this visible PL emission can be identified easily with the naked eye. Therefore, $\mathrm{ZnS}$ :Mn NPs are suitable candidate for potential applications in optoelectronic and biophotonic devices. Particularly, water-soluble $\mathrm{ZnS}$ :Mn can be used for fluorescence imaging in living tissues. Some recent studies have reported enhanced three-photon as well as four-photon absorption properties of ZnS:Mn NPs which can make them useful for imaging applications because infrared (IR) wavelength is converted to a wavelength in the blue or UV region and this increases the resolution beyond the diffraction limit. However, water-soluble $\mathrm{Mn}^{2+}$-doped $\mathrm{ZnS}$ (ZnS:Mn) NPs synthesized by the presented method may be useful in the applications as biolabels, etc. due to their nanometric dimensions and visible luminescent properties combined with their multiphoton absorption properties. However, the synthesized NPs need to be functionalized with suitable materials for making them bioactive and to retain their size and shape after they are tagged with biomolecules.

Acknowledgments Authors are grateful to Department of Science and Technology (SR/FTP/PS-67/2008), Government of India, for the financial support. A.K.K. is grateful to Ministry of Human Resource Development, National Institute of Technology Durgapur, India, for the maintenance scholarship. Authors are thankful to the referee for providing valuable advice in improving the manuscript.

Open Access This article is distributed under the terms of the Creative Commons Attribution License which permits any use, distribution and reproduction in any medium, provided the original author(s) and source are credited.

\section{References}

Akihito F, Hideo W, Ken-Ichiro S, Shigeru N, Masato H (2000) Diamond-ZnS composite infrared window. Proc SPIE 4375: 206-217. doi:10.1117/12.439177

Bhargava RN (1996) Doped nanocrystalline materials-physics and applications. J Lumin 70:85-94. doi:10.1016/0022-2313(96) 00046-4

Bhargava RN, Gallagher D, Hong X, Nurmikko A (1994) Optical properties of manganese-doped nanocrystals of $\mathrm{ZnS}$. Phys Rev Lett 72:416-419. doi:10.1103/PhysRevLett.72.416

Brus L (1998) Electronic wave functions in semiconductor clusters: experiment and theory. J Phys Chem 90:2555-2560. doi: 10.1021/j100403a003

Cao J, Yang J, Zhang Y, Yang L, Wang Y, Wei M, Liuc Y, Gao M, Liu X, Xie Z (2009) Optimized doping concentration of manganese in zinc sulfide nanoparticles for yellow-orange light 
emission. J Alloy Compd 486:890-894. doi:10.1016/j.jallcom. 2009.07.097

Chattopadhyay M, Kumbhakar P, Sarkar R, Mitra AK (2009a) Enhanced three-photon absorption and nonlinear refraction in $\mathrm{ZnS}$ and $\mathrm{Mn}^{2+}$ doped $\mathrm{ZnS}$ quantum dots. Appl Phys Lett 95:163115. doi:10.1063/1.3254186

Chattopadhyay M, Kumbhakar P, Tiwary CS, Sarkar R, Mitra AK, Chatterjee U (2009b) Multiphoton absorption and refraction in $\mathrm{Mn}^{2+}$ doped ZnS quantum dots. J Appl Phys 105:024313. doi: 10.1063/1.3072678

Chen W, Li G, Malm J, Huang Y, Wallenberg R, Han H, Wang Z, Bovin J (2000) Pressure dependence of $\mathrm{Mn}^{2+}$ fluorescence in ZnS: $\mathrm{Mn}^{2+}$ nanoparticles. J Lumin 91:139-145. doi:10.1016/S00 22-2313(00)00222-2

Cullity BD (1978) Elements of X-ray diffraction, 2nd edn. Addison Wesley, USA

Denzler D, Olschewski M, Sattler K (1998) Luminescence studies of localized gap states in colloidal ZnS nanocrystals. J Appl Phys 84:2841-2845. doi:10.1063/1.368425

Dong B, Cao L, Su G, Liu W, Qu H, Zhai H (2010) Water-soluble $\mathrm{ZnS}: \mathrm{Mn} / \mathrm{ZnS}$ core/shell nanoparticles prepared by a novel twostep method. J Alloy Compd 492:363-367. doi:10.1016/ j.jallcom.2009.11.096

Fang X, Bando Y, Gautam UK, Zhai T, Zeng H, Xu X, Liao M, Golberg D (2004) ZnO and ZnS nanostructures: ultraviolet-light emitters, lasers, and sensors. Crit Rev Solid State 34:190-223. doi:10.1080/10408430903245393

Fayaz M, Tiwary CS, Kalaichelvan PT, Venkatesan R (2010) Blue orange light emission from biogenic synthesized silver nanoparticles using Trichoderma viride. Colloid Surfaces B 75:175-178. doi:10.1016/j.colsurfb.2009.08.028

Goldman ER, Balighian ED, Kuno MK, Labrenz S, Tran PT, Anderson GP, Mauro JM, Mattoussi J (2002) Luminescent quantum dotadaptor protein-antibody conjugates for use in fluoroimmunoassays. Phys Status Solidi B 229:407-414. doi:10.1002/15213951(200201)229:1<407:AID-PSSB407>3.0.CO;2-S

$\mathrm{Hu}$ H, Zhang W (2006) Synthesis and properties of transition metals and rare-earth metals doped $\mathrm{ZnS}$ nanoparticles. Opt Mater 28:536-550. doi:10.1016/j.optmat.2005.03.015

Karar N, Singh F, Mehata BR (2004) Structure and photoluminescence studies on ZnS:Mn nanoparticles. J Appl Phys 95:656660. doi:10.1063/1.1633347

Kripal R, Gupta AK, Mishra SK, Srivastava RK, Pandey AC, Prakash SG (2010) Photoluminescence and photoconductivity of $\mathrm{ZnS}: \mathrm{Mn}^{2+}$ nanoparticles synthesized via co-precipitation method. Spectrochim Acta A 76:523-530. doi:10.1016/j.saa. 2010.04.018

Lu SW, Lee BI, Wang ZL, Tong W, Wagner BK, Park W, Summers CJ (2001) Synthesis and photoluminescence enhancement of $\mathrm{Mn}^{2+}$ doped ZnS nanocrystals. J Lumin 92:73-78. doi:10.1016/ S0022-2313(00)00238-6

Lu X, Yang J, Fu Y, Liu Q, Qi B, Lu C, Su Z (2010) White light emission from $\mathrm{Mn}^{2+}$ doped $\mathrm{ZnS}$ nanocrystals through the surface chelating of 8-hydroxyquinoline-5-sulfonic acid. Nanotechnology 21:115702. doi:10.1088/0957-4484/21/11/115702
Murugadoss G, Rajamannan B, Ramasamy V (2010) Synthesis, characterization and optical properties of water-soluble $\mathrm{ZnS}: \mathrm{Mn}^{2+}$ nanoparticles. J Lumin 130:2032-2039. doi:10.1016/j.jlumin.2010. 05.022

Nakada T, Furumi K, Kunioka A (1999) High-efficiency cadmiumfree $\mathrm{Cu}(\mathrm{In}, \mathrm{Ga}) \mathrm{Se}_{2}$ thin-film solar cells with chemically deposited ZnS buffer layers. IEEE T Electron Dev 46:2093-2097. doi: 10.1109/16.792002

Nazerdeylami S, Saievar-Iranizad E, Dehghani Z, Molaei M (2011) Synthesis and photoluminescent and nonlinear optical properties of manganese doped ZnS nanoparticles. Physica B 406:108-111. doi:10.1016/j.physb.2010.10.033

Pankove JI (1971) Optical processes in semiconductor. Prentice-Hall, New Jersey

Peng WQ, Qu SC, Cong GW, Zhang XQ, Wang ZG (2005) Concentration effect of $\mathrm{Mn}^{2+}$ on the photoluminescence of ZnS:Mn nanocrystals. J Cryst Growth 279:454-460. doi: 10.1016/j.jcrysgro.2005.05.005

Peng WQ, Cong GW, Qu SC, Wang ZG (2006) Synthesis and photoluminescence of $\mathrm{ZnS}: \mathrm{Cu}$ nanoparticles. Opt Mater 29:313-317. doi:10.1016/j.optmat.2005.10.003

Pradhan N, Goorskey D, Thessing J, Peng X (2005) An alternative of CdSe nanocrystal emitters: pure and tunable impurity emissions in ZnSe nanocrystals. J Am Chem Soc 127:17586-17587. doi: $10.1021 / \mathrm{ja} 055557 \mathrm{z}$

Ren Z, Yang H, Shen L, Han SD (2008) Hydrothermal preparation and properties of nanocrystalline ZnS:Mn. J Mater Sci Mater Electron 19:1-4. doi:10.1007/s10854-007-9340-3

Sapra S, Prakash A, Ghangrekar A, Periasamy N, Sharma DD (2005) Emission properties of manganese-doped $\mathrm{ZnS}$ nanocrystals. J Phys Chem B 109:1663-1668. doi:10.1021/jp049976e

Sarkar R, Tiwari CS, Kumbhakar P, Basu S, Mitra AK (2008) Yellow-orange light emission from $\mathrm{Mn}^{2+}$ doped $\mathrm{ZnS}$ nanoparticles. Physica E 40:3115-3120. doi:10.1016/j.physe.2008.04.013

Son D, Jung D, Kim J, Moon T, Kim C, Park B (2007) Synthesis and photoluminescence of Mn-doped zinc Sulfide nanoparticles. Appl Phys Lett 90:101910. doi:10.1063/1.2711709

Sooklal K, Cullum BS, Angel SM, Murphy CJ (1996) Photophysical properties of $\mathrm{ZnS}$ nanoclusters with spatially localized $\mathrm{Mn}^{2+}$. J Phys Chem 100:4551-4555. doi:10.1021/jp952377a

Tiwari CS, Kumbhakar P, Mondal AK, Mitra AK (2010) Synthesis and enhanced green photoluminescence emission from BCT ZnS nanocrystals. Phys Status Solidi A 207:1874-1879. doi:10.1002/ pssa.200925341

Xiao Q, Xiao C (2008) Synthesis and photoluminescence of watersoluble $\mathrm{Mn}^{2+}$-doped $\mathrm{ZnS}$ quantum dots. Appl Surf Sci 254:6432-6435. doi:10.1016/j.apsusc.2008.04.002

Yang H, Holloway PH, Ratna BB (2003) Photoluminescent and electroluminescent properties of Mn-doped $\mathrm{ZnS}$ nanocrystals. J Appl Phys 93:586-592. doi:10.1063/1.1529316

Ye C, Fang X, Li G, Zhang L (2004) Origin of the green photoluminescence from zinc sulfide nanobelts. Appl Phys Lett 85:3035-3037. doi:10.1063/1.1807018 